\title{
The b-Chromatic Number of Graphs
}

\author{
K.P.Thilagavathy \\ Assistant Professor \\ Kumaraguru College of Technology \\ Coimbatore
}

\author{
A.Santha \\ Associate Professor \\ Sri Sakthi Institute of Engineering \& Technology \\ Coimbatore
}

\begin{abstract}
The purpose of this study is to compute the b-chromatic number of central graph of star graph $K_{1, n}$ and Multi star Graphs $K_{2}\left(a_{n}, a_{s}\right)$ and $K_{3}\left(a_{n}, a_{r}, a_{t}\right)$.More Specifically, for any star graph $\varphi\left[C\left(K_{1, n}\right)\right]=n$, for any double star graph $\varphi\left[C\left(K_{2}\left(a_{n}, a_{r}\right)\right)\right]=n+r+1$ and for the triple star graph, $\varphi\left[C\left(K_{3}\left(a_{n}, a_{r}, a_{t}\right)\right)\right]=n+r+t+1$. Also find out the bchromatic number of central graph of Fire cracker graph and Banana tree graph.
\end{abstract}

\section{Keywords}

Central graph, b-chromatic number, Star graph, Double Star graph, Firecracker graph, Banana tree

\section{INTRODUCTION}

The b-chromatic number $[1,4,6], \varphi(G)$ of a graph $G$ is the maximum number of colours for which $G$ has a proper colouring such that every colour class contains a vertex adjacent to a vertex of every other colour.

For a given graph of $G=(V, E)$ we do an operation on $G$, by subdividing each edge exactly once and joining all the non adjacent vertices of $G$.The graph obtained by this process is called a Central Graph[9,10] of $G$ denoted by $C(G)$.All graphs here are undirected.

A complete bipartite graph $K_{1, n}$ is called a star graph [2].

The multi star graph [7] $K_{m}\left(a_{n}, a_{r}, \ldots, a_{s}\right)$ is formed by joining $a_{n}, a_{r}, \ldots, a_{s}$ end-edges to the $m$-nodes of $K_{m}$.

\section{THE B-COLOURING OF CENTRAL GRAPH OF STAR GRAPH AND MULTI STAR GRAPH}

\subsection{Theorem}

For any star graph $K_{1, n}$, the b-chromatic number $\varphi\left[C\left(K_{1, n}\right)\right]=n, n>1$

\subsection{Example}

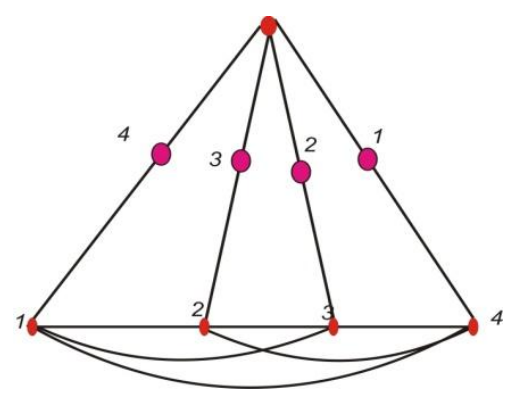

Fig1: $\varphi\left[C\left(K_{1,4}\right)\right]=4$

\subsection{Result}

For any double star graph $K_{2}\left(a_{n}, a_{r}\right)$ the bchromatic number $\varphi\left[C\left(K_{2}\left(a_{n}, a_{r}\right)\right)\right]=n+r+1$

\subsection{Example}

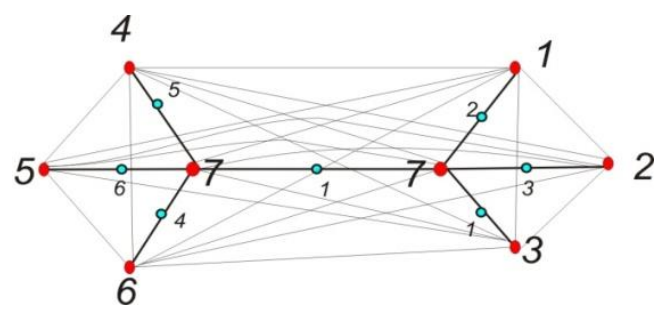

Fig 2: $\varphi\left[C\left(K_{2}\left(a_{3}, a_{3}\right)\right)\right]=7$

\subsection{Result}

For any triple star graph $K_{3}\left(a_{n}, a_{r}, a_{t}\right)$, the bchromatic number $\varphi\left[C\left(K_{3}\left(a_{n}, a_{r}, a_{t}\right)\right)\right]=n+r+t+1$ 


\section{THE B-COLOURING OF CENTRAL GRAPH OF FIRECRACKER GRAPH 3.1 Fire cracker graph}

An $(n, k)$-fire cracker[3] is a graph obtained by the concatenation of $n$ copies of $k$-stars by linking one leaf from each and it is denoted by $F_{n, k}$.

\subsection{Example}

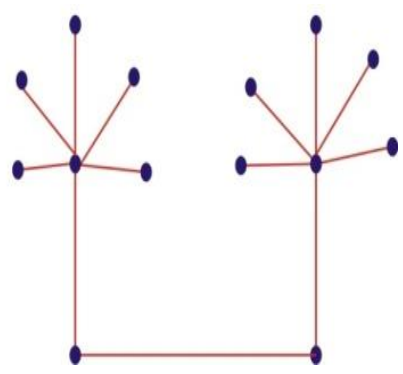

Fig-3: $F_{2,6}$

\subsection{Structural Properties of Fire graph}

- Number of edges in the graph $F_{n, k}$ is $q=m n$

- Number of vertices in the graph $F_{n, k}$ is $p=m n-1$

- Maximum degree in the graph $F_{n, k}$ is $\Delta=m$

- Number of vertices in the graph $C F_{n, k}$ is $p_{C(B m, n)}=2 m n+1$

- Maximum degree in the graph $C F_{n, k}$ is $\Delta_{C(B m, n)}=m n$

\subsection{Theorem}

For any fire cracker graph, the b-chromatic number $\varphi\left[C\left(F_{2, n}\right)\right]=2 n-2, \quad n>2$

Proof Let $G=F_{2, n}$ be the fire cracker graph. By definition, a $(2, n)$ fire cracker graph is obtained by concatenation of $2, n$-stars by linking one leaf from each. Let the two stars be denoted as $S^{1} \& S^{2}$. Let $V=\left\{v_{1}, v_{2}, . ., v_{n}\right\}$ be the vertex set of $S^{1}$ and $U=\left\{u_{1}, u_{2}, \ldots, u_{n}\right\}$ be the vertex set of $S^{2}$.Now the vertex arrangement of $S^{1} \& S^{2}$ are as follows: Name the star $S^{1}$ by the vertices in $V$ in the anti clock wise sense and $S^{2}$ by the vertices in $U$ in the same anti clock direction, where $u_{n} \& v_{n}$ are the root vertices and let $u_{n-1} \& v_{n-1}$ are the bridge. Now in $C\left[F_{2, n}\right]$, let $v_{i j}$ be the newly introduced vertex in the edge joining $v_{i} \& v_{j}$. These stars $S^{1} \& S^{2}$ becomes the complete graph $K_{n-1}^{i}, i=1,2$. Now assign a colouring to $C\left[F_{2, n}\right]$ as follows. Assign the colour $C_{i}$ to $v_{i}$, $1 \leq i \leq n-1$ and $C_{i}{ }^{1}$ to $u_{i}, 1 \leq i \leq n-1$. There are $2(n-1)$ colours are needed to colour the complete graphs $K^{1}{ }_{n-1} \& K^{2}{ }_{n-1}$.Suppose we want to introduce a new colour say $C_{n}$.we have to use this new colour to one of the root vertex $u_{n}$ or $v_{n}$.Then this $C_{n}$ is adjacent only with $n-1$ colours. So that, we cant introduce a colour. Therefore the maximum possible colouring is $2(n-1)$.

$$
\begin{aligned}
& \text { Hence } \varphi\left[C\left(F_{2, n}\right)\right]=n-1+n-1 \\
& =2 n-2, n=3,4, \ldots .
\end{aligned}
$$

\subsection{Example}

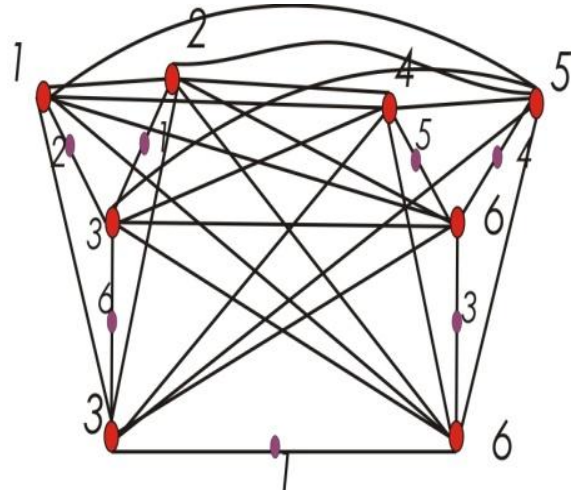

Fig-4: $\varphi\left[C\left(F_{2,4}\right)\right]=6$

\section{THE B-COLOURING OF CENTRAL GRAPH OF BANANA TREE GRAPH 4.1 Banana tree}

A $(m, n)$ banana tree [3] is a graph obtained by connecting one leaf of each $m$-copies of a $n$-star graph with a single root vertex.

\subsection{Structural Properties of Banana tree graph}

- Number of edges in the graph $B_{m, n}$ is $q=m n$

- $\quad$ Number of vertices in the graph $B_{m, n}$ is $p=m n+1$

- Maximum degree in the graph $B_{m, n}$ is $\Delta=m$

- Number of vertices in the graph $C B_{m, n}$ is $p_{C(B m, n)}=2 m n+1$

- Maximum degree in the graph $C B_{m, n}$ is $\Delta_{C(B m, n)}=m n$ 


\subsection{Theorem}

For any banana tree $B_{m, n}$, the b-chromatic number $\varphi\left[C\left(B_{m, n}\right)\right]=m n-m+1, \quad m, n \geq 2$

\subsection{Example}

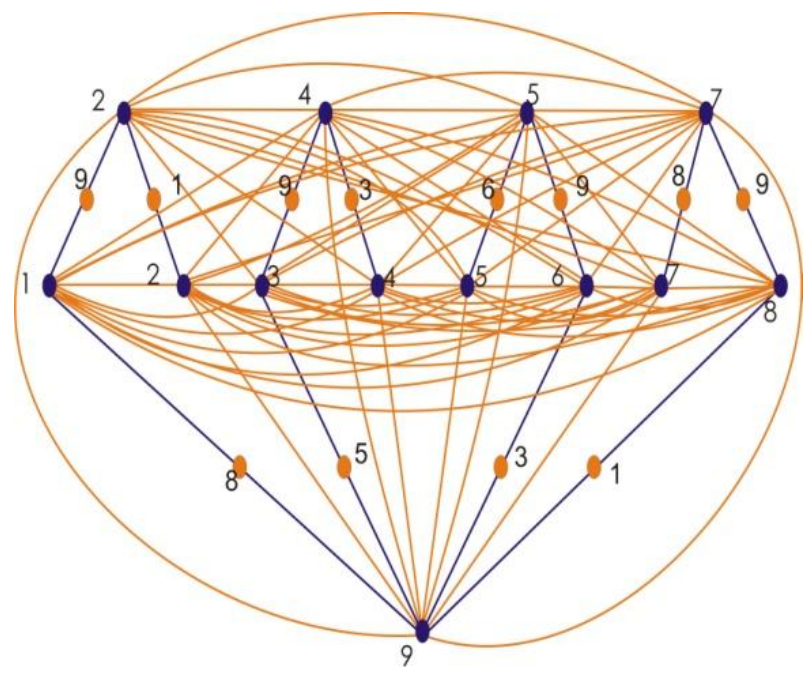

Fig-5: $\varphi\left[C\left(B_{4,3}\right)\right]=9$

\section{CONCLUSION}

In this paper we have determined the b-Chromatic number of Star graphs and some star related graphs. This study can be extended for the achromatic number of all the above mentioned graphs. And the comparison of these two colorings on the star graph family is under study.

\section{REFERENCES}

[1] Brice Effantin and Hamammache Kheddouchi, "The $b$ chromatic number of some power graphs", Discrete Mathematics and Theoretical Computer Science, 46-54, 2003.

[2] Chen, W. C.; Lü, H. I.; and Yeh, Y. N. "Operations of Interlaced Trees and Graceful Trees." South East Asian Bull. Math. 21, 337-348, 1997.

[3] Gallian, J. "Dynamic Survey of Graph Labeling." Elec. J. Combin. 14, No. DS 6 , Jan. 3, 2007.

[4] Irving, R.W and Manlove, D.F, "The b-Chromatic number of a graph", Discrete Applied Mathematics 91(1-3) 127$141,1999$.

[5] Jonathan gross, Jay yellan ,"Hand book of graph theory"CRC press,Newyork,2004

[6] Marko Jakovac, Sandi Klavzar "The b-chromatic number of cubic Graphs", Graphs and Combinatories 26:107$118,2010$.

[7] Nikipoulos,S.D and Rondogiannis ,P. "On the Number of Spanning trees of Multistar Related Graphs", Volume 65,Issue 4,183-188,1998.

[8] Thilagavathi, K. Thilagavathy,K.P. Roopesh, N. "The achromatic colouring of graphs" Electronic Notes in Discrete Mathematics 33,153-156, 2009.

[9] Thilagavathi, K. Vijayalakshmi,D. Roopesh, N. "Bcolouring of Central Graphs" International Journal of Computer Applications(0975-8887)Vol.3,No.11, July 2010.

[10] Vernold Vivin, J. Thilagavathi, K. "On Harmonious colouring of Central Graphs", Far East. Math. Sci(FJMS), 2, pp189-197,2006.

[11] Vernold Vivin,J. Thilagavathi, K. "On Harmonious colouring of Line graph of path", Applied Mathematical sciences, Vol.3,no.5,205-214,2009. 